\title{
Existence and Uniqueness of Uncertain Fractional Backward Difference Equations of Riemann-Liouville Type
}

\author{
Pshtiwan Othman Mohammed $\mathbb{D}^{1},{ }^{1}$ Thabet Abdeljawad $\left(\mathbb{D},{ }^{2,3,4}\right.$ Fahd Jarad $\left(\mathbb{D},{ }^{5}\right.$ \\ and Yu-Ming Chu $\mathbb{1}^{6,7}$ \\ ${ }^{1}$ Department of Mathematics, College of Education, University of Sulaimani, Kurdistan Region, Sulaimani, Iraq \\ ${ }^{2}$ Department of Mathematics and General Sciences, Prince Sultan University, P.O. Box 66833, Riyadh 11586, Saudi Arabia \\ ${ }^{3}$ Department of Medical Research, China Medical University, Taichung 40402, Taiwan \\ ${ }^{4}$ Department of Computer Science and Information Engineering, Asia University, Taichung, Taiwan \\ ${ }^{5}$ Department of Mathematics, Cankaya University, Etimesgut, Ankara 06790, Turkey \\ ${ }^{6}$ Department of Mathematics, Huzhou University, Huzhou 313000, China \\ ${ }^{7}$ Hunan Provincial Key Laboratory of Mathematical Modeling and Analysis in Engineering, \\ Changsha University of Science \& Technology, Changsha 410114, China
}

Correspondence should be addressed to Thabet Abdeljawad; tabdeljawad@psu.edu.sa and Yu-Ming Chu; chuyuming@zjhu.edu.cn

Received 6 August 2020; Revised 6 September 2020; Accepted 29 September 2020; Published 17 October 2020

Academic Editor: A. M. Abd-Alla

Copyright ( $) 2020$ Pshtiwan Othman Mohammed et al. This is an open access article distributed under the Creative Commons Attribution License, which permits unrestricted use, distribution, and reproduction in any medium, provided the original work is properly cited.

In this article, we consider the analytic solutions of the uncertain fractional backward difference equations in the sense of Riemann-Liouville fractional operators which are solved by using the Picard successive iteration method. Also, we consider the existence and uniqueness theorem of the solution to an uncertain fractional backward difference equation via the Banach contraction fixed-point theorem under the conditions of Lipschitz constant and linear combination growth. Finally, we point out some examples to confirm the validity of the existence and uniqueness of the solution.

\section{Introduction}

Fractional calculus is based on an old idea that has become important and popular in applications only recently. The idea is to generalize integration and differentiation to noninteger orders in order to develop and extend the theory of calculus and to describe a more extensive range of possible doings in reality. During the past decades, fractional differential equations have been widely employed in many fields: mathematical analysis, optics and thermal systems, control engineering, and robotics, see, for example, [1-9].

In recent years, uncertain fractional differential and difference equations and discrete difference equations have become popular in both theory and applications. These represent a new area for researchers which was developed slowly in their early stages. By using modeling techniques with discrete fractional calculus, some researchers established the existence, uniqueness, monotonicity, multiplicity, and qualitative properties of solutions to uncertain fractional difference equations (UFDEs) in the sense of Riemann-Liouville, Caputo, and $\mathrm{AB}$ operators; for further details, see [10-22] and the references cited therein.

The aim of this attempt is to investigate the existence and uniqueness of fractional difference equations in the sense of Riemann-Liouville-like difference operator with assuming Lipschitz condition on its nonlinear term. Our findings are partial continuation of some results obtained in [23-25]. It is worth mentioning that the uncertainty theory of fractional difference equations is used to make the problems have a unique solution almost surely. 


\section{Preliminaries}

This section presents some preliminaries, definitions, and facts in the field of uncertainty theory and discrete fractional calculus, see, e.g., [12, 26-28]. Throughout the article, we consider $\mathbb{N}_{a}=\{a, a+1, a+2, \ldots$,$\} for a \in \mathbb{R}$ and the backward jump operator $\rho(r):=r-1$ for $r \in \mathbb{N}_{a}$.

Definition 1 (see [26]). For any function $f: \mathbb{N}_{a} \longrightarrow \mathbb{R}$, the backward difference operator is defined by

$$
\nabla f(t)=f(t)-f(\rho(t)), \quad t \in \mathbb{N}_{a},
$$

while the backward sum is given by

$$
\nabla_{a}^{-1} f(t)=\sum_{r=a+1}^{t} f(r), \quad t \in \mathbb{N}_{a+1} .
$$

Definition 2 (see [26-29]). For any natural number $j$, the $\nabla$-rising factorial function of $t$ is defined by

$$
t^{\bar{j}}=\prod_{\ell=0}^{j-1}(t+\ell), \quad t^{\overline{0}}=1
$$

Moreover, for any $v \in \mathbb{R}$, the $\nabla$-rising factorial function is defined by

$$
t^{\bar{\nu}}=\frac{\Gamma(t+\nu)}{\Gamma(t)}, \quad 0^{\bar{\nu}}=0
$$

for $t \in \mathbb{R} \backslash\{\ldots,-2,-1,0\}$. Also, note that the division by negative integer poles of the gamma function gives zero.

A major property of the rising factorial function is as follows:

$$
\nabla\left(t^{\bar{\nu}}\right)=v t^{\overline{\nu-1}}
$$

This implies that $t^{\bar{\nu}}$ is increasing on $\mathbb{N}_{0}$ such that $\nu>0$.

Definition 3 (see [13, 14, 26-29]). For any function $f: \mathbb{N}_{a} \longrightarrow \mathbb{R}$, the nabla fractional sum of order $v>0$ in the sense of Riemann-Liouville is defined by

$$
\begin{aligned}
& \left(\nabla_{a}^{-v} f\right)(t)=\frac{1}{\Gamma(\nu)} \sum_{r=a+1}^{t}(t-\rho(r))^{\overline{\nu-1}} f(r), \\
& \left(\nabla_{a}^{0} f\right)(t)=f(t) .
\end{aligned}
$$

Lemma 1 (see $[13,14,26-28])$. For any function $f$ defined on $\mathbb{N}_{a}$ and any $\nu, \alpha>0$, we have

$$
\left(\nabla_{a}^{-\gamma} \nabla_{a}^{-\alpha} f\right)(t)=\left(\nabla_{a}^{-(\alpha+v)} f\right)(t)=\left(\nabla_{a}^{-\alpha} \nabla_{a}^{-\nu} f\right)(t) .
$$

Lemma 2 (see $[13,14,26-28]$ ). For any function $f$ defined on $\mathbb{N}_{a}$ and any $v>0$, we have

$$
\left(\nabla_{a}^{v} f\right)(t)=\left(\nabla \nabla_{a}^{-(1-v)} f\right)(t)
$$

Lemma 3 (see $[13,14,26-28]$ ). For any function $f$ defined on $\mathbb{N}_{a}$ and any $v>0$, we have

$$
\left(\nabla_{a}^{-\nu} \nabla f\right)(t)=\left(\nabla \nabla_{a}^{-\nu} f\right)(t)-\frac{(t-a)^{\overline{\nu-1}}}{\Gamma(\nu)} f(a)
$$

Lemma 4 (see $[13,14,26-28])$. For any function $f$ defined on $\mathbb{N}_{a}$ and any $v>0$, we have

$$
\begin{aligned}
& \left(\nabla_{a}^{-v} \nabla^{v} f\right)(t)=\left(\nabla_{a}^{\nu} \nabla^{-v} f\right)(t)=f(t), \quad v \notin \mathbb{N}, \\
& \left(\nabla_{a}^{-v} \nabla^{v} f\right)(t)=f(t)-\sum_{k=0}^{\nu-1} \frac{(t-a)^{\bar{k}}}{k !} \nabla^{k} f(a), \quad v \in \mathbb{N} .
\end{aligned}
$$

Lemma 5 (see $[13,14,26-28]$ ). For any $a \in \mathbb{R}$ and $\nu, \alpha>0$, we have

$$
\nabla_{a}^{-\nu}(t-a)^{\bar{\alpha}}=\frac{\Gamma(\alpha+1)}{\Gamma(\alpha+\nu+1)}(t-a)^{\overline{\alpha+\nu}} .
$$

Motivated by the definition of the $n$ th-order backward sum for uncertain sequence $\xi_{t}$, we define the $\nu$ th-order backward sum for uncertain sequence $\xi_{t}$ as follows:

Definition 4 (see $[13,14,28]$ ). Let $v>0, a \in \mathbb{R}$, and $\xi_{t}$ be an uncertain sequence indexed by $t \in \mathbb{N}_{a}$. Then, we have

$$
\nabla_{a}^{-v} \xi_{t}=\frac{1}{\Gamma(\nu)} \sum_{r=a+1}^{t}(t-\rho(r))^{\overline{\nu-1}} \xi_{r}
$$

which is called the $v$ th-order backward fractional sum of uncertain sequence $\xi_{t}$.

Definition 5 (see $[13,14,28])$. For any $v>0$, the fractional Riemann-Liouville-like backward difference for uncertain sequence $\xi_{t}$ is defined by

$$
\nabla_{a}^{v} \xi_{t}=\nabla_{a}\left(\nabla_{a}^{-(1-v)} \xi_{t}\right)
$$

Next, we recall the definition of nabla discrete MittagLeffler (ML).

Definition 6 (see [28]). For any $\lambda \in \mathbb{R}$ and $\nu, \mu, \eta \in \mathbb{C}$ with $\operatorname{Re}(\nu)>0$, the two-parameter discrete ML function is defined by

$$
E_{\overline{\nu, \mu}}(\lambda, \eta):=\sum_{\ell=0}^{\infty} \lambda^{\ell} \frac{\eta^{\overline{\ell \nu+\mu-1}}}{\Gamma(\ell \nu+\mu)}, \quad|\lambda|<1
$$

Particularly, if $\mu=1$, we get the one-parameter discrete ML function:

$$
E_{\bar{\nu}}(\lambda, \eta):=\sum_{\ell=0}^{\infty} \lambda^{\ell} \frac{\eta^{\overline{\ell v}}}{\Gamma(\ell \nu+1)}, \quad|\lambda|<1 .
$$




\section{UFBDE and Existence and Uniqueness Theorem}

First, we recall the inverse uncertainty distribution theory.

Definition 7. (see [11]). An uncertainty distribution $\Psi$ is called regular if it is a continuous and strictly increasing function and satisfies

$$
\begin{aligned}
& \lim _{x \longrightarrow-\infty} \Psi(x)=0, \\
& \lim _{x \longrightarrow+\infty} \Psi(x)=1 .
\end{aligned}
$$

Definition 8. (see [11]). Let $\xi$ be an uncertain variable with a regular uncertainty distribution $\Psi$. Then, the inverse function $\Psi^{-1}$ is called the inverse uncertainty distribution of $\xi$.

Example 1. From Definition 8, we deduce that the following:

(i) The inverse uncertainty distribution of a linear uncertain variable $\mathscr{L}(a, b)$ is given by

$$
\Psi^{-1}(\alpha)=(1-\alpha) a+\alpha b
$$

(ii) The inverse uncertainty distribution of a normal uncertain variable $\mathscr{L}(e, \sigma)$ is given by

$$
\Psi^{-1}(\alpha)=e+\frac{\sqrt{3} \sigma}{\pi} \ln \left(\frac{\alpha}{1-\alpha}\right) .
$$

(iii) The inverse uncertainty distribution of a normal uncertain variable $\mathscr{L} \mathscr{O} \mathscr{G} \mathcal{N}(e, \sigma)$ is given by

$$
\Psi^{-1}(\alpha)=\exp (e)+\left(\frac{\alpha}{1-\alpha}\right)^{(\sqrt{3} \sigma / \pi)} .
$$

Definition 9. (see [11]). We say that an uncertain variable $\xi$ is symmetrical if

$$
\Psi(x)+\Psi(-x)=1
$$

where $\Psi(x)$ is a regular uncertainty distribution of $\xi$.

Remark 1. From Definition 9, we can deduce that the symmetrical uncertain variable has the inverse uncertainty distribution $\Psi^{-1}(\alpha)$ that satiates

$$
\Psi^{-1}(\alpha)+\Psi^{-1}(1-\alpha)=0
$$

Example 2. From Definition 9, we deduce that the following:

(1) The linear uncertain variable $\mathscr{L}(-a, a)$ is symmetrical for any positive real number $a$

(2) The normal uncertain variable $\mathscr{L}(0,1)$ is symmetrical
Definition 10. (see [11]; i.i.d. definition). In statistics and probability theory, a collection of random variables $\xi_{i} s$ is independent and identically distributed (or briefly, i.i.d.) if each random variable $\xi_{i}$ has the same probability distribution as the others and all are mutually independent.

Then, we state the definition of the UFBDE.

Definition 11. An uncertain fractional difference equation is a fractional difference equation which is driven by an uncertain sequence. Moreover, an uncertain fractional backward difference equation for the Riemann-Liouville type is the uncertain fractional difference equation with Riemann-Liouville-like backward difference.

Consider the following generalized Riemann-Liouville fractional difference equation:

$$
\left(\nabla_{\alpha-1}^{\alpha} y\right)(t)=G(t, y(t))+H(t, y(t)) \xi_{t},
$$

subject to the initial condition (i.c.)

$$
\left.\left(\nabla_{\alpha-1}^{-(1-\alpha)} y\right)(t)\right|_{t=\alpha}=y(\alpha)
$$

where $\nabla_{\alpha-1}^{\alpha}$ denotes fractional Riemann-Liouville-like backward difference with $0<\alpha<1, G, H$ are two real-valued functions defined on $[1, \infty] \times \mathbb{R}, t \in \mathbb{N}_{1} \cap[1, T+1]$, $y(\alpha) \in \mathbb{R}$ is a crisp number, and $\xi_{1}, \xi_{2}, \ldots, \xi_{T+1}$ are $(T+1)$-i.i.d. uncertain variables with symmetrical uncertainty distribution $\mathscr{L}(a, b)$.

Remark 2. Observe that the i.i.d. uncertain variables are those uncertain variables that are independent and have the same uncertainty distribution. See [11] for more detail.

Lemma 6. Initial value problem (22) with i.c. (23) is equivalent to the following uncertain fractional sum equation:

$$
\begin{aligned}
y(t)= & \frac{(t-\alpha+1)^{\overline{\alpha-1}}}{\Gamma(\alpha)} y(\alpha) \\
& +\frac{1}{\Gamma(\alpha)} \sum_{r=\alpha+1}^{t}(t-\rho(r))^{\overline{\alpha-1}}\left[G(r, y(r))+H(r, y(r)) \xi_{r}\right],
\end{aligned}
$$

for $t \in \mathbb{N}_{\alpha+1} \cap[1, T+1]$.

Proof. The proof is very similar to [29], Lemma 5.1, and [30], Theorem 2, hereby applying the operator $\nabla_{\alpha}^{-\alpha}$ to IVP (22) with Definition 3, Lemma 2, and Lemma 3, so we omit this.

In this paper, the following special linear UFBDE will be considered:

$$
\begin{aligned}
\nabla_{\alpha-1}^{\alpha} y(t) & =\lambda y(t)+\lambda \xi_{t}, \\
\left.\nabla_{\alpha-1}^{-(1-\alpha)} y(t)\right|_{t=\alpha} & =y(\alpha),
\end{aligned}
$$

for $t \in \mathbb{N}_{1} \cap[1, T+1], n \in \mathbb{N}_{1}$, and $\lambda \in(0,1)$.

Remark 3. The following identity is useful in proving the upcoming theorem. From Definition 3 and Lemma 5, we can deduce for any real number $a$ 


$$
\begin{aligned}
\nabla_{a}^{-\alpha}(t-a+1)^{\bar{\beta}}= & \frac{\Gamma(\beta+1)}{\Gamma(\alpha+\beta+1)}(t-a+1)^{\overline{\alpha+\beta}} \\
& -\frac{\Gamma(\beta+1)}{\Gamma(\alpha)}(t-a+1)^{\overline{\alpha-1}},
\end{aligned}
$$

where $\alpha>0$ and $\beta>-1$.

Theorem 1. For any $t \in \mathbb{N}_{\alpha+1} \cap[1, T+1]$ and $|\lambda|<1$, linear UFBDE (25) with the initial condition (26) has a solution

$$
y(t)=(1-\lambda) E_{\overline{\alpha, \alpha}}(\lambda, t-\alpha+1) y(\alpha)+\xi_{t},
$$

where $\xi_{t}$ is an uncertain sequence with the uncertainty distribution $\mathscr{L}\left(a \cdot \lambda E_{\overline{\alpha, \alpha+1}}(\lambda, t-\alpha), b \cdot \lambda E_{\overline{\alpha, \alpha+1}}(\lambda, t-\alpha)\right)$.

Proof. Applying the operator $\nabla_{\alpha}^{-\alpha}$ to equation (25), we get

$$
\begin{array}{r}
\left.\nabla_{\alpha}^{-\alpha}\left(\nabla_{\alpha-1}^{\alpha} y(t)\right)=\lambda \nabla_{\alpha}^{-\alpha} y(t)\right)+\lambda \nabla_{\alpha}^{-\alpha} \xi_{t}, \\
t \in \mathbb{N}_{\alpha+1} \cap[1, T+1] .
\end{array}
$$

Making use of Lemma 2 and Lemma 3 to the left-hand side of (29), we get

$$
\begin{aligned}
& \nabla_{\alpha}^{-\alpha}\left(\nabla_{\alpha-1}^{\alpha} y(t)\right)=\nabla_{\alpha}^{-\alpha}\left(\nabla_{\alpha} \nabla_{\alpha-1}^{-(1-\alpha)} y(t)\right) \\
&=\nabla_{\alpha}\left(\nabla_{\alpha}^{-\alpha} \nabla_{\alpha-1}^{-(1-\alpha)} y(t)\right)-\frac{(t-\alpha+1)^{\overline{\alpha-1}}}{\Gamma(\alpha)} y(\alpha), \\
&=y(t)-\frac{(t-\alpha+1)^{\overline{\alpha-1}}}{\Gamma(\alpha)} y(\alpha), \\
& t \in \mathbb{N}_{\alpha+1} \cap[1, T+1]
\end{aligned}
$$

It follows from this and equation (29) that

$$
\begin{array}{r}
y(t)=\frac{(t-\alpha+1)^{\overline{\alpha-1}}}{\Gamma(\alpha)} y(\alpha)+\lambda \nabla_{\alpha}^{-\alpha} y(t)+\lambda \nabla_{\alpha}^{-\alpha} \xi_{t}, \\
t \in \mathbb{N}_{\alpha+1} \cap[1, T+1],
\end{array}
$$

which is the solution of UFBDE (28).

To derive the solution, we use the Picard approximation recurrence formula with a starting point $y_{0}(t)=$ $\left((t-\alpha+1)^{\frac{\alpha-1}{\alpha-1}} / \Gamma(\alpha)\right) y(\alpha)$ for each $t \in \mathbb{N}_{\alpha-1} \cap[1, T+1]$. The other components can be determined by using the following recurrence relation:

$$
y_{j}(t)=\frac{(t-\alpha+1)^{\overline{\alpha-1}}}{\Gamma(\alpha)} y(\alpha)+\lambda \nabla_{\alpha}^{-\alpha} y_{j-1}(t)+\lambda \nabla_{\alpha}^{-\alpha} \xi_{t},
$$

for $t \in \mathbb{N}_{\alpha+1} \cap[1, T+1]$ and $j \in \mathbb{N}_{1}$. Since $\xi_{1}, \xi_{2}, \ldots, \xi_{T+1}$ are i.i.d. uncertain variables, we write $\xi_{t}=\xi$ in distribution. By using Lemma 5, Remark 3, and the fact that the linear combination of finite independent uncertain variables is an uncertain variable with a positive linear combination coefficient (see Theorems 1.21-1.24 of [11]), we can deduce

$$
\begin{aligned}
y_{1}(t)= & \frac{(t-\alpha+1)^{\overline{\alpha-1}}}{\Gamma(\alpha)} y(\alpha)+\lambda \nabla_{\alpha}^{-\alpha} y_{0}(t)+\lambda \nabla_{\alpha}^{-\alpha} \xi \\
= & \frac{(t-\alpha+1)^{\overline{\alpha-1}}}{\Gamma(\alpha)} y(\alpha) \\
& +\lambda\left[\frac{(t-\alpha+1)^{\overline{2 \alpha-1}}}{\Gamma(2 \alpha)}-\frac{(t-\alpha+1)^{\overline{\alpha-1}}}{\Gamma(\alpha)}\right] y(\alpha)
\end{aligned}
$$$$
+\lambda \frac{(t-\alpha)^{\bar{\alpha}}}{\Gamma(\alpha+1)} \xi
$$$$
y_{2}(t)=\frac{(t-\alpha+1)^{\overline{\alpha-1}}}{\Gamma(\alpha)} y(\alpha)+\lambda \nabla_{\alpha}^{-\alpha} y_{1}(t)+\lambda \nabla_{\alpha}^{-\alpha} \xi
$$$$
=\frac{(t-\alpha+1)^{\overline{\alpha-1}}}{\Gamma(\alpha)} y(\alpha)
$$$$
+\lambda\left[\frac{(t-\alpha+1)^{\overline{2 \alpha-1}}}{\Gamma(2 \alpha)}-\frac{(t-\alpha+1)^{\overline{\alpha-1}}}{\Gamma(\alpha)}\right] y(\alpha)
$$$$
+\lambda^{2}\left[\frac{(t-\alpha+1)^{\overline{3 \alpha-1}}}{\Gamma(3 \alpha)}-\frac{(t-\alpha+1)^{\overline{2 \alpha-1}}}{\Gamma(2 \alpha)}\right] y(\alpha)
$$$$
+\left[\frac{\lambda(t-\alpha)^{\bar{\alpha}}}{\Gamma(\alpha+1)}+\frac{\lambda^{2}(t-\alpha)^{\overline{2 \alpha}}}{\Gamma(2 \alpha+1)}\right] \xi
$$

and so on, continuing the process up to the $j$ th term to get

$$
\begin{aligned}
y_{j}(t)= & \sum_{k=0}^{j} \lambda^{k}\left[\frac{(t-\alpha+1)^{\overline{k \alpha+\alpha-1}}}{\Gamma((k+1) \alpha)}-\frac{(t-\alpha+1)^{\overline{k \alpha-1}}}{\Gamma(k \alpha)}\right] y(\alpha) \\
& +\sum_{k=1}^{j} \lambda^{k} \frac{(t-\alpha)^{\overline{k \alpha}}}{\Gamma(k \alpha+1)} \xi,
\end{aligned}
$$

for each $t \in \mathbb{N}_{\alpha+1} \cap[1, T+1]$. Observe that the two series 


$$
\begin{aligned}
\sum_{k=0}^{\infty} \lambda^{k}\left[\frac{(t-\alpha+1)^{\overline{k \alpha+\alpha-1}}}{\Gamma((k+1) \alpha)}-\frac{\left.(t-\alpha+1)^{\overline{k \alpha-1}}\right]}{\Gamma(k \alpha)}\right] & =E_{\overline{\alpha, \alpha}}(\lambda, t-\alpha+1)-\lambda E_{\overline{\alpha, \alpha}}(\lambda, t-\alpha+1) \\
& =(1-\lambda) E_{\overline{\alpha, \alpha}}(\lambda, t-\alpha+1), \\
\sum_{k=1}^{\infty} \lambda^{k} \frac{(t-\alpha)^{\overline{k \alpha}}}{\Gamma(k \alpha+1)} & =\lambda E_{\overline{\alpha, \alpha+1}}(\lambda, t-\alpha),
\end{aligned}
$$

are absolutely convergent for $|\lambda|<1$ by the d'Alembert ratio comparison test, and the limitation $\bar{Y}(t):=\lim _{j \rightarrow \infty} y_{j}$ exists. Thus, we have

$$
\begin{array}{r}
\bar{Y}(t)=\lambda E_{\overline{\alpha, \alpha+1}}(\lambda, t-\alpha) \xi+(1-\lambda) E_{\overline{\alpha, \alpha}}(\lambda, t-\alpha+1) y(\alpha), \\
t \in \mathbb{N}_{\alpha+1} \cap[1, T+1] .
\end{array}
$$
yields

On the contrary, taking the limit on both sides of (32)

$$
\begin{array}{r}
\bar{Y}(t)=\frac{(t-\alpha+1)^{\overline{\alpha-1}}}{\Gamma(\alpha)} y(\alpha)+\lambda \nabla_{\alpha}^{-\alpha} \bar{Y}(t)+\lambda \nabla_{\alpha}^{-\alpha} \xi_{t}, \\
t \in \mathbb{N}_{\alpha+1} \cap[1, T+1] .
\end{array}
$$

That is, $\bar{Y}(t)$ satisfies equation (31), and hence, $\bar{Y}(t)$ is a solution of equation (25) subject to the initial condition (26). Thus, our proof is completed.

The following theorem provides and confirms the existence and uniqueness of the solution of UFBDEs.

Theorem 2. Assume that $G(t, x)$ and $H(t, x)$ satisfy the Lipschitz condition

$$
|G(t, x)-G(t, y)|+|H(t, x)-H(t, y)| \leq L|x-y|,
$$

and there is a positive number $L$ that satisfies the following inequality:

$$
L<\frac{\Gamma(\alpha+1) \Gamma(T+1-\alpha)}{\Gamma(T+1)(Q+1)},
$$

where $Q=|a| \vee|b|$. Then, UFBDE (25) subject to the initial condition (26) has a unique solution $y(t)$ for $t \in \mathbb{N}_{\alpha+1} \cap[1, T+1]$ almost surely.

Proof. Define

$$
\begin{aligned}
l_{\alpha}^{k} & :=\left\{x ; x=\{x(t)\}_{\alpha+1}^{k}, k \in \mathbb{N}_{1}\right\},\|x\|, \\
& :=\max _{t \in \mathbb{N}_{\alpha+1} \cap[1, T+1]}|x(t)|,
\end{aligned}
$$

where $\{x(t)\}_{\alpha}^{k}$ are finite real sequences which have $k$ terms. It is clear that $\left(l_{\alpha}^{k},\|\cdot\|\right)$ is a Banach space (see [31], Chapter 4). Now, for any $y_{t} \in l_{\alpha}^{k}$, we define the operator $P$ as follows:

$$
\begin{aligned}
P y_{t}= & \frac{(t-\alpha)^{\overline{\alpha-1}}}{\Gamma(\alpha)} y(\alpha)+\frac{1}{\Gamma(\alpha)} \sum_{r=\alpha+1}^{t}(t-\rho(r))^{\overline{\alpha-1}}\left[G\left(r, y_{r}\right)\right. \\
& \left.+H\left(r, y_{r}\right) \xi_{r}\right] .
\end{aligned}
$$

Since $\xi_{t}\left(t \in \mathbb{N}_{\alpha+1} \cap[1, T+1]\right)$ is an uncertain variable at each time $t$ with the linear uncertainty distribution $\mathscr{L}(a, b)$, we have $\mathscr{M}\left\{\left(\xi_{t}<a\right) \cup\left(\xi_{t}>b\right)\right\}=0$. The inequality $\xi_{t}(\gamma) \leq Q$ (where $Q=|a| \vee|b|$ ) holds almost surely for any $\gamma \in \chi\left\{\left(\xi_{t}<a\right) \cup\left(\xi_{t}>b\right) ; t \in \mathbb{N}_{\alpha+1} \cap[1, T+1]\right\}$, where $\chi$ represents the universal set on the uncertainty space. Then, by making use of the assumptions and Lemma 5, we have, for any $x_{t}, y_{t} \in l_{\alpha}^{k}$, 


$$
\begin{aligned}
& \left\|P x_{t}(\gamma)-P y_{t}(\gamma)\right\|=\max _{t \in \mathbb{N}_{\alpha+1} \cap[1, T+1]}\left|P x_{t}(\gamma)-P y_{t}(\gamma)\right|, \\
& \leq \frac{1}{\Gamma(\alpha)} \max _{t \in \mathbb{N}_{\alpha+1} \cap[1, T+1]} \sum_{r=\alpha+1}^{t}(t-\rho(r))^{\overline{\alpha-1}} \\
& \times\left(\mid G\left(r, x_{r}(\gamma)-G\left(r, y_{r}(\gamma)|+|\left[H\left(r, x_{r}(\gamma)-H\left(r, y_{r}(\gamma)\right] \xi_{r} \mid\right),\right.\right.\right.\right. \\
& \leq \frac{1}{\Gamma(\alpha)} \max _{t \in \mathbb{N}_{\alpha+1} \cap[1, T+1]} \sum_{r=\alpha+1}^{t}(t-\rho(r))^{\overline{\alpha-1}} \\
& \times\left(\mid G\left(r, x_{r}(\gamma)-G\left(r, y_{r}(\gamma)|+Q| H\left(r, x_{r}(\gamma)-H\left(r, y_{r}(\gamma) \mid\right),\right.\right.\right.\right. \\
& \leq L(1+Q) \frac{1}{\Gamma(\alpha)} \max _{t \in \mathbb{N}_{\alpha+1} \cap[1, T+1]} \sum_{r=\alpha+1}^{t}(t-\rho(r))^{\overline{\alpha-1}}\left|x_{r}(\gamma)-y_{r}(\gamma)\right|, \\
& \leq L(1+Q)\left\|x_{t}(\gamma)-y_{t}(\gamma)\right\| \max _{t \in \mathbb{N}_{\alpha+1} \cap[1, T+1]}\left(\nabla_{\alpha}^{-\alpha}(t-\alpha)^{\overline{0}}\right) \\
& =L(1+Q)\left\|x_{t}(\gamma)-y_{t}(\gamma)\right\| \max _{t \in \mathbb{N}_{\alpha+1} \cap[1, T+1]}\left(\frac{1}{\Gamma(\alpha+1)}(t-\alpha)^{\bar{\alpha}}\right) \text {, } \\
& \leq \frac{L(1+Q)(T+1-\alpha)^{\bar{\alpha}}}{\Gamma(\alpha+1)}\left\|x_{t}(\gamma)-y_{t}(\gamma)\right\| \\
& =\frac{L(1+Q) \Gamma(T+1)}{\Gamma(\alpha+1) \Gamma(T-\alpha+1)}\left\|x_{t}(\gamma)-y_{t}(\gamma)\right\| .
\end{aligned}
$$

Now, we can observe that the mapping $P$ is a contraction in $l_{\alpha}^{k}$ almost surely with $0<L<(\Gamma(\alpha+1) \Gamma(T-\alpha+1) /(1+$ Q) $\Gamma(T+1))($ see [31], Chapter 4$)$. Then, by using the Banach contraction mapping theorem (see [31], Chapter 4), we get a unique fixed point $y_{t}(\gamma)$ of $P$ in $l_{\alpha}^{k}$ almost surely. Moreover, $y_{t}(\gamma)=\lim _{j \rightarrow \infty} y_{t}^{j}(\gamma)$, where $y_{t}^{j}(\gamma)=P\left(y_{t}^{j-1}(\gamma)\right)$, with $y_{t}^{0}(\gamma)=\left((t-\alpha)^{\overline{\alpha-1}} / \Gamma(\alpha)\right) y(\alpha)$.

For any given $t \in \mathbb{N}_{\alpha+1} \cap[1, T+1]$, as $G$ and $H$ are Lipschitz continuous functions, the operator $P$ is measurable. Since $y_{t}^{1}(\gamma), y_{t}^{2}(\gamma), \ldots, y_{t}^{j}(\gamma), \ldots$ are uncertain variables and $y_{t}^{0}(\gamma)$ is a real-valued measurable function of uncertain variables, $y_{t}^{0}(\gamma)$ is an uncertain variable by [11], Theorem 1.10. Hence, $y_{t}=\lim _{j \longrightarrow \infty} y_{t}^{j}$ is an uncertain variable (see [21], Theorem 3).

Consequently, UFBDE (25) with i.c. (26) has a unique solution $y_{t}$ for $t \in \mathbb{N}_{\alpha+1} \cap[1, T+1]$ almost surely.

\section{Example Illustrations}

This section deals with some examples to confirm the validity of Theorem 2 .
Example 3. Consider the following UFBDE:

$$
\nabla_{(-1 / 2)}^{(1 / 2)} y(t)=\left\{\begin{array}{l}
\frac{\ln (|y(t)|+1)}{2 t^{3}}+0.5 \xi_{t}, \quad t \in \mathbb{N}_{1} \cap[1,4], \\
y\left(\frac{1}{2}\right)=1,
\end{array}\right.
$$

where $\xi_{1}, \ldots, \xi_{4}$ are four i.i.d. uncertain variables with uncertainty distribution $\mathscr{L}(-1,2)$.

According to Lemma 6 with $\alpha=(1 / 2)$, the inverse uncertainty distribution of the solution for UFBDE (43) is the solution of the following sum equation:

$$
\begin{aligned}
y(t)= & \frac{(t+(1 / 2))^{\overline{-0.5}}}{\Gamma(0.5)} y(\alpha)+\frac{1}{\Gamma(0.5)} \sum_{r=(3 / 2)}^{t}(t-\rho(r))^{\overline{-0.5}} \\
& \cdot\left(\frac{\ln (|y(r)|+1)}{4 r^{3}}+0.25 \xi_{r}\right), \quad t \in \mathbb{N}_{0.5} \cap[1,4] .
\end{aligned}
$$


Then, for $t \in \mathbb{N}_{0.5} \cap[1,4]$, we have

$$
\begin{aligned}
|G(t, x)-G(t, y)|+|H(t, x)-H(t, y)| & =\left|\frac{\ln (|x|+1)}{2 t^{3}}-\frac{\ln (|y|+1)}{2 t^{3}}\right|, \\
& =\frac{1}{2 t^{3}}|\ln (|x|+1)-\ln (|y|+1)|, \\
& \leq \frac{1}{2(3 / 2)^{3}}|| x|-| y|| \leq \frac{4|x-y|}{27}, \\
\frac{\Gamma(0.5+1) \Gamma(4+1-0.5)}{3 \Gamma(4+1)} & \approx 0.1636>\frac{4}{27}=0.1481 .
\end{aligned}
$$

Thus, UFBDE (43) has a unique solution almost surely by Theorem 2 .

Example 4. We consider the following UFBDE:

$$
\nabla_{-0.25}^{0.25} y(t)=\frac{y^{2} t}{40}+\xi_{t}, \quad t \in \mathbb{N}_{1} \cap[1,4],
$$

where $\xi_{1}, \xi_{2}, \xi_{3}, \xi_{4}$ are four i.i.d. linear uncertain variables with linear uncertainty distribution $\mathscr{L}(-3,3)$.

According to Lemma 6 with $\alpha=(1 / 4)$, the inverse uncertainty distribution of the solution for UFBDE (46) is the solution of the following sum equation:

$$
\begin{aligned}
y(t)= & \frac{(t+(1 / 2))^{\overline{-(3 / 4)}}}{\Gamma(0.25)} y(\alpha) \\
& +\frac{1}{\Gamma(0.25)} \sum_{r=1.25}^{t}(t-\rho(r))^{\overline{-(3 / 4)}}\left(\frac{y^{2} r}{40}+\xi_{r}\right) .
\end{aligned}
$$

Observe that $|G(t, x)-G(t, y)|+|H(t, x)-H(t, y)|$ is Lipschitz-continuous in $[-20,20]$ with Lipschitz constant 0.1 as follows:

$$
\begin{aligned}
& |G(t, x)-G(t, y)|+|H(t, x)-H(t, y)| \leq \frac{1}{40}|x+y||x-y| \\
& \quad=0.1|x-y| .
\end{aligned}
$$

Also, we have

$$
\frac{\Gamma(0.25+1) \Gamma(3+1-0.25)}{4 \Gamma(3+1)} \approx 0.167>0.1 .
$$

Consequently, UFBDE (50) has a unique solution almost surely by Theorem 2 .

Example 5. Consider the following UFBDE:

$$
\nabla_{-0.5}^{0.5} y(t)=\frac{\sin (t y)}{10+t^{2}}+0.1 \xi_{t}, \quad t \in \mathbb{N}_{1} \cap[1,4],
$$

where $\xi_{1}, \xi_{2}, \xi_{3}, \xi_{4}$ are 4 i.i.d. linear uncertain variables with linear uncertainty distribution $\mathscr{L}(-1,1)$.
According to Lemma 6 with $\alpha=(1 / 2)$, the inverse uncertainty distribution of the solution for UFBDE (50) is the solution of the following sum equation:

$$
\begin{aligned}
y(t)= & \frac{(t+(1 / 2))^{\overline{-0.5}}}{\sqrt{\pi}} y(\alpha) \\
& +\frac{1}{\sqrt{\pi}} \sum_{r=1.5}^{t}(t-\rho(r))^{\overline{-0.5}}\left(\frac{\sin (r y)}{10+r^{2}}+0.1 \xi_{r}\right) .
\end{aligned}
$$

Then, we can directly verify that

$$
\begin{aligned}
|G(t, x)-G(t, y)|+|H(t, x)-H(t, y)| & \leq \frac{1}{10}|x-y|, \\
\frac{\Gamma(0.5+1) \Gamma(3+1-0.5)}{2 \Gamma(3+1)} & \approx 0.2454>\frac{1}{10}=0.1 .
\end{aligned}
$$

Consequently, UFBDE (50) has a unique solution almost surely by Theorem 2 .

\section{Conclusion}

We have presented analytical solutions to a special type of linear UFBDEs. Moreover, a Lipschitz condition with its constant is given to provide a unique solution almost surely to an UFBDE. It can be seen that our obtained results pave the way for the future works, that is, to investigate the stability analysis and applications of UFBDEs.

\section{Data Availability}

No data were used to support this study.

\section{Conflicts of Interest}

The authors declare that they have no conflicts of interest.

\section{Authors' Contributions}

All authors contributed equally and significantly to writing this article. All authors read and approved the final manuscript. 


\section{Acknowledgments}

The first author would like to thank Prince Sultan University for funding this work through research group Nonlinear Analysis Methods in Applied Mathematics (NAMAM) (group number: RG-DES-2017-01-17).

\section{References}

[1] K. S. Miller and B. Ross, An Introduction to the Fractional Calculus and Fractional Differential Equations, John Wiley \& Sons, New York, NY, USA, 1993.

[2] I. Podlubny, Fractional Differential Equations, Academic Press, San Diego, CA, USA, 1999.

[3] A. A. Kilbas, H. M. Srivastava, and J. J. Trujillo, Theory and Applications of Fractional Differential Equations, Elsevier B.V., Amsterdam, Netherlands, 2006.

[4] K. Diethelm, The Analysis of Fractional Differential Equations, Springer, Berlin, Germany, 2010.

[5] F. K. Hamasalh and P. O. Muhammad, "Generalized quartic fractional spline interpolation with applications," International Journal of Open Problems in Computer Science and Mathematics, vol. 8, no. 1, pp. 67-80, 2015.

[6] F. K. Hamasalh and P. O. Muhammed, "Computational method for fractional differential equations using nonpolynomial fractional spline," Mathematical Sciences Letters, vol. 5, no. 2, pp. 131-136, 2016.

[7] R. Almeida, N. R. O. Bastos, and M. T. T. Monteiro, "Modeling some real phenomena by fractional differential equations," Mathematical Methods in the Applied Sciences, vol. 39, no. 16, pp. 4846-4855, 2016.

[8] M. Martinez, P. O. Mohammed, and J. E. N. Valdes, "Nonconformable fractional Laplace transform," Kragujevac Journal of Mathematics, vol. 46, no. 3, pp. 341-354, 2022.

[9] P. O. Mohammed and M. Z. Sarikaya, "On generalized fractional integral inequalities for twice differentiable convex functions," Journal of Computational and Applied Mathematics, vol. 372, Article ID 112740, 2020.

[10] M. Bohner and A. C. Peterson, Advances in Dynamic Equations on Time Scales, Birkhauser, Boston, MA, USA, 2003.

[11] B. Liu, Uncertainty Theory: A Branch of Mathematics for Modeling Human Uncertainty, Springer, Berlin, Germany, 2010.

[12] C. Goodrich and A. Peterson, Discrete Fractional Calculus, Springer, Berlin, Germany, 2015.

[13] F. Atici and P. Eloe, "A transform method in discrete fractional calculus,” IJDE, vol. 2, no. 2, pp. 165-176, 2007.

[14] F. Atici and P. Eloe, "Initial value problems in discrete fractional calculus," Proceedings of the American Mathematical Society, vol. 137, no. 3, pp. 981-989, 2009.

[15] C. S. Goodrich, "Existence of a positive solution to a system of discrete fractional boundary value problems," Applied Mathematics and Computation, vol. 217, no. 9, pp. 4740-4753, 2011.

[16] G.-C. Wu and D. Baleanu, "Discrete chaos in fractional delayed logistic maps," Nonlinear Dynamics, vol. 80, no. 4, pp. 1697-1703, 2015.

[17] G.-C. Wu, D. Baleanu, and W.-H. Luo, "Lyapunov functions for Riemann-Liouville-like fractional difference equations," Applied Mathematics and Computation, vol. 314, pp. 228-236, 2017.
[18] I. Suwan, T. Abdeljawad, and F. Jarad, "Monotonicity analysis for nabla $h$-discrete fractional Atangana-Baleanu differences," Chaos, Solitons \& Fractals, vol. 117, pp. 50-59, 2018.

[19] T. Abdeljawad and D. Baleanu, "Monotonicity analysis of a nabla discrete fractional operator with discrete Mittag-Leffler kernel," Chaos Solitons \& Fractals, vol. 116, pp. 1-5, 2017.

[20] Y. Zhu, "Uncertain fractional differential equations and an interest rate model," Mathematical Methods in the Applied Sciences, vol. 38, no. 15, pp. 3359-3368, 2015.

[21] Y. Zhu, "Existence and uniqueness of the solution to uncertain fractional differential equation," Journal of Uncertainty Analysis and Applications, vol. 3, pp. 1-11, 2015.

[22] Z. Lu and Y. Zhu, "Numerical approach for solution to an uncertain fractional differential equation," Applied Mathematics and Computation, vol. 343, pp. 137-148, 2019.

[23] J. W. He, L. Zhang, Y. Zhou, and B. Ahmad, "Existence of solutions for fractional difference equations via topological degree methods," Advances in Difference Equations, vol. 2018, p. $153,2018$.

[24] P. O. Mohammed, "A generalized uncertain fractional forward difference equations of Riemann-Liouville type," Journal of Mathematics Research, vol. 11, no. 4, pp. 43-50, 2019.

[25] Q. Lu, Y. Zhu, and Z. Lu, "Uncertain fractional forward difference equations for Riemann-Liouville type," Advances in Difference Equations, vol. 2019, p. 147, 2019.

[26] T. Abdeljawad, "On delta and nabla caputo fractional differences and dual identities," Discrete Dynamics in Nature and Society, vol. 2013, Article ID 406910, 12 pages, 2013.

[27] T. Abdeljawad, F. Jarad, and J. Alzabut, "Fractional proportional differences with memory," The European Physical Journal Special Topics, vol. 226, no. 16-18, pp. 3333-3354, 2017.

[28] T. Abdeljawad, "Different type kernel $h$-fractional differences and their fractional $h$-sums," Chaos, Solitons \& Fractals, vol. 116, pp. 146-156, 2018.

[29] T. Abdeljawad and F. M. Atici, "On the definitions of nabla fractional operators," Abstract and Applied Analysis, vol. 2012, Article ID 406757, 13 pages, 2012.

[30] F. Atici and P. Eloe, "Gronwall's inequality on discrete fractional calculus," Computers \& Mathematics with Applications, vol. 64, pp. 3193-3200, 2012.

[31] P. Sacks, Techniques of Functional Analysis for Differential and Integral Equations, Academic Press, London, UK, 2017. 